\title{
Collecting Histories of Education and Employment During Recovery (CHEER) Project
}

\section{What are we hoping to learn?}

The goal of the Collecting Histories and Employment during Recovery (CHEER) study is to provide a longterm picture of career development activities of young adults (YA) living with serious mental health conditions (SMHCs). From this study, we will better understand the following from YA with SMHCs:

- Their work and education experiences;

- The supports and barriers encountered when trying to achieve work and/or education goals; and

- How becoming a parent has impacted their work and education experiences.

Our study is grounded in participatory action research principles, where all activities are guided and informed by the active participation of YA with lived experience of mental health conditions in all phases of this study.

\section{Why is this study important?}

Work and school experiences in early adulthood predict later career success. ${ }^{1}$ However, YA living with SMHCs have low employment and educational attainment rates. ${ }^{2,}$ ${ }^{3,4}$ Lack of educational attainment and career exploration in YA with SMHCs can lead to little or no economic progress later in life. ${ }^{5}$

In addition, YA with SMHCs are more likely to become first-time parents at an earlier age than their peers without SMHCs. ${ }^{6,7} 8$ Research suggests that YA who become parents when they are under the age of 27 are likely to be unemployed or under-employed in shortterm, part-time, low-paying jobs. ${ }^{9}$

\section{Who is involved in this study?}

CHEER aims to recruit 60 YA with SMHCs. Forty participants will be YA who are not parents, between the ages of 25-30. Twenty participants will be young parents between the ages 22-30, who have been parenting for one year or more. All participants must meet the following criteria:

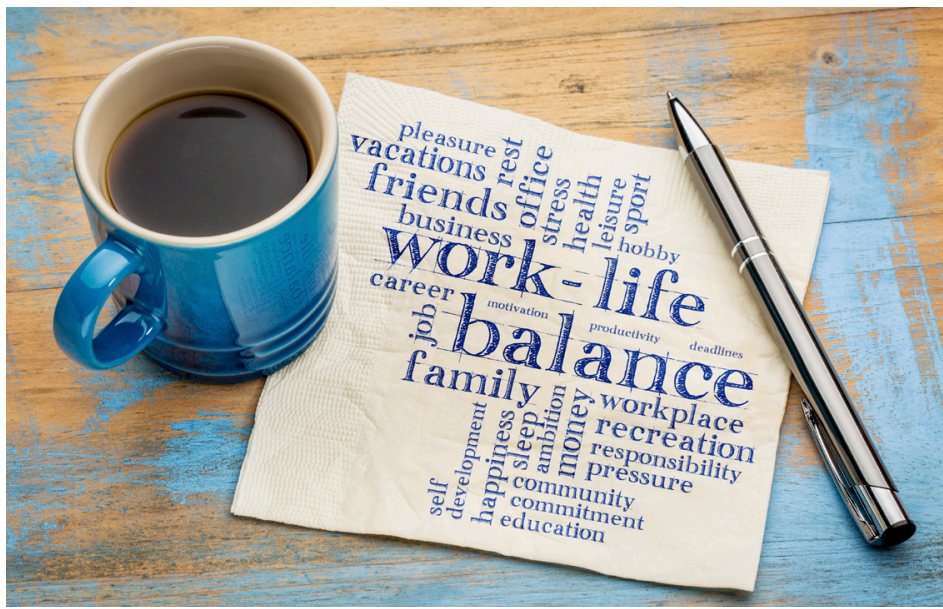

- Have been diagnosed with a SMHC (e.g., Major Depression Disorder, Anxiety Disorder, Schizophrenia, etc.) before the age of 22;

- Have some post high school employment and/or education experience; and

- Have a history of one or more of the following:

- Overnight hospitalization;

- Partial hospitalization;

- Being a client of/receiving services from the Massachusetts Department of Mental Health;

- Receiving special education services; or

- Needing to take a formal leave of absence from school and/or employment due to their mental health condition.

\section{What does the study involve?}

Participants will take part in a 90 minute one-time, inperson interview that focuses on past and current education, training, and work experiences - both successes and challenges. The interview also includes questions about how other parts of the participant's life influences work and education experiences, such as:

- General and family background;

- Mental health and physical health;

- Major life events; and 
- Parenting and child rearing (if applicable).

All participants will receive a $\$ 30$ gift card for their participation.

\section{What are the anticipated outcomes}

After data analysis is complete, we will be able to:

- Provide a descriptive picture of the typical education, employment, and job training goals and experiences of YA with SMHCs;

- Identify key targets for future services and interventions for YA with SMHCs that contribute to school and work success and alleviate challenges they face; and

- Assist service providers in developing improved support systems for young parents with SMHCs and their families.

We will use study findings to inform the development and delivery of services that support the school and work experiences of young adults, including young parents, with SMHCs.

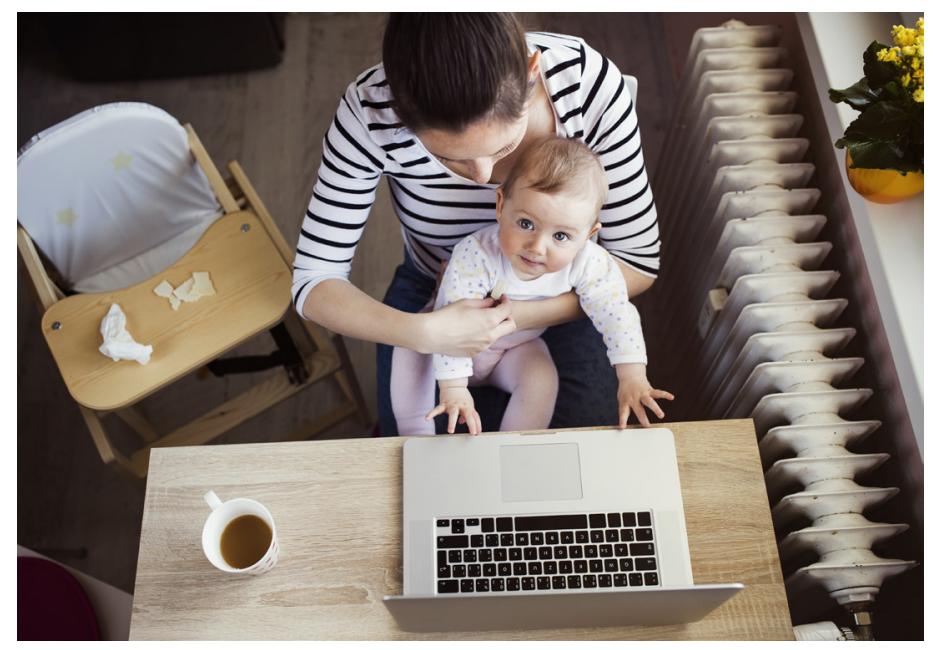

\section{References}

1. De Vos, A., Clippeleer, I., \& Dewilde, T. (2009). Proactive career behaviours and career success during the early career. Journal of Occupational and Organizational Psychology, 82(4), 761-777. doi:10.1348/096317909X471013

2. Newman, L., Wagner, M., Knokey, A.-M., Marder, C., Nagle, K., Shaver, D., Wei, X., with Cameto, R., Contreras, E., Ferguson, K., Greene, S., and Schwarting, M. (2011). The post-high school outcomes of young adults with disabilities up to 8 years after high school. A report from the national longitudinal transition study-2 (NLTS2) (NCSER 2011-3005). Menlo Park, CA: SRI International.

3. Salzer, M. S., Wick, L. C., \& Rogers, J. A. (2008). Familiarity with and use of accommodations and supports among postsecondary students with mental illnesses. Psychiatric Services, 59(4), 370-375. doi:10.1176/appi.ps.59.4.370

4. Armstrong, K. H., Dedrick, R. F., \& Greenbaum, P. E. (2003). Factors associated with community adjustment of young adults with serious emotional disturbance a longitudinal analysis. Journal of Emotional and Behavioral Disorders, 11(2), 66-76. doi:10.1177/106342660301100201

5. Baron, R. C., \& Salzer, M. S. (2002). Accounting for unemployment among people with mental illness. Behavioral Sciences \& the Law, 20(6), 585-599. doi:10.1002/bsl.513

6. GAO. (2008). Young adults with serious mental illness: Some states and federal agencies are taking steps to address their transition challenges (GAO-08-678). Washington, D.C.: United States Government Accountability Office.

7. Nicholson, J. (2014). For parents with mental health conditions: The chance to "have it all" at work and at home. Psychiatric Rehabilitation Journal, 37(2), 153-156. doi:10.1037/prj0000057

8. Stykes J. (2011). Fatherhood in the U.S.: Men's age at first birth, 1987-2010 (FP-11-04). Retrieved from National Center for Family \& Marriage Research: http://www.bgsu.edu/ content/dam/BGSU/college-of-arts-and-sciences/NCFMR/ documents/FP/FP-11-04.pdf

9. Osgood, D. W., Ruth, G., Eccles, J. S., Jacobs, J. E., \& Barber, B. L. (2005). Six paths to adulthood: Fast starters, parents without careers, educated partners, educated singles, working singles, and slow starters. Chicago, IL: The University of Chicago Press. doi:10.7208/chicago/9780226748924.003.0010

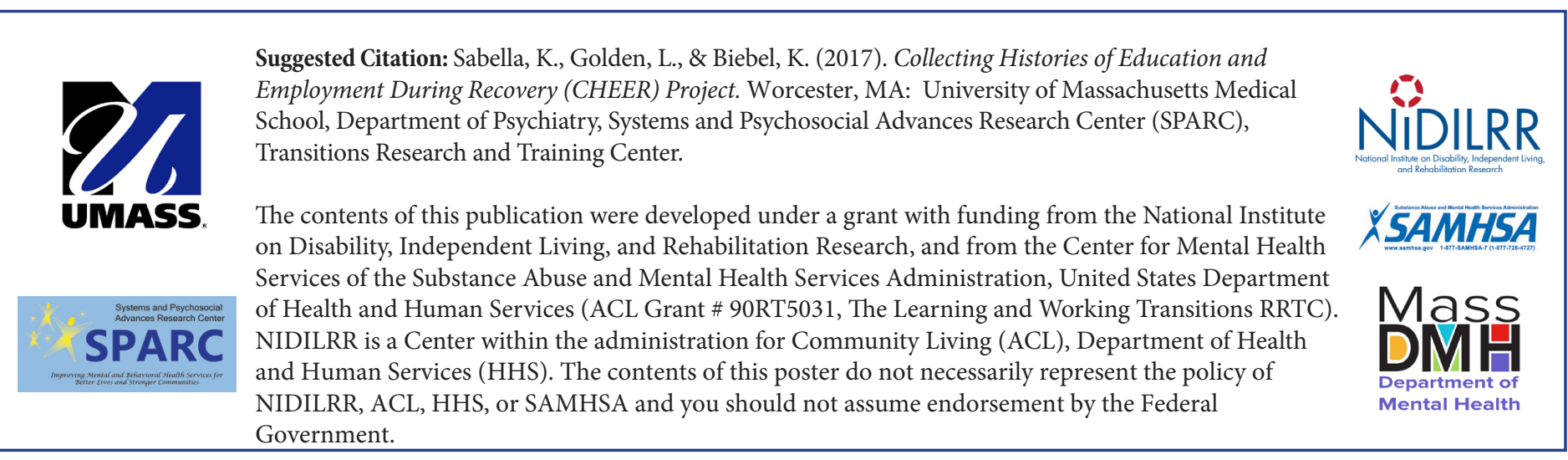

This is a product of Psychiatry Information in Brief. An electronic copy of this issue with full references can be found at http://escholarship.umassmed.edu/pib/vol14/iss4/1 